\title{
Low-repetition-rate femtosecond operation in extended-cavity mode-locked Yb:CALGO laser
}

\author{
D. N. Papadopoulos, ${ }^{1, *}$ F. Druon, ${ }^{1}$ J. Boudeile,${ }^{1}$ I. Martial, ${ }^{1}$ M. Hanna, ${ }^{1}$ P. Georges, ${ }^{1}$ \\ P. O. Petit, ${ }^{2}$ P. Goldner, ${ }^{2}$ and B. Viana ${ }^{2}$ \\ ${ }^{1}$ Laboratoire Charles Fabry de l'Institut d'Optique, CNRS, Université Paris Sud, RD 128, 91127 Palaiseau, France \\ ${ }^{2}$ Laboratoire de Chimie de la matière condensée de Paris (UMR 7574), \\ 11 Rue Pierre et Marie Curie 75231 Paris \\ *Corresponding author: dimitris.papadopoulos@institutoptique.fr
}

Received October 24, 2008; revised December 1, 2008; accepted December 1, 2008; posted December 10, 2008 (Doc. ID 103232); published January 14, 2009

We report on an extended-cavity mode-locked laser based on an Yb:CALGO crystal operating either at $27 \mathrm{MHz}$ and $93 \mathrm{fs}$ pulse duration or at $15 \mathrm{MHz}$ and $170 \mathrm{fs}$ duration single-pulse regime. To the best of our knowledge this is the first demonstration of an extended-cavity oscillator based on Yb-doped crystal producing sub-100 fs pulses. The pulse energy was $24 \mathrm{~nJ}$ directly at the output of the oscillator (and $17 \mathrm{~nJ}$ after compression). Based on a similar design, we also demonstrate an unprecedented double-pulse dualwavelength femtosecond regime. An explanation of this atypical regime is proposed. () 2009 Optical Society of America

OCIS codes: $140.3615,140.4050$.

In the field of femtosecond lasers, an intense interest has been shown for ytterbium-doped laser crystals. These crystals are well known to be particularly suitable for very efficient, directly diode-pumped, solidstate femtosecond oscillators. However, it has been shown that the spectral properties of the $\mathrm{Yb}^{3+}$ dopant strongly depend on the matrix host, and a lot of work has been done to find the perfect matrix to allow the development of both ultrashort and high-power lasers. In fact, broadband emission spectrum and good thermal conductivity are often antagonistic for rareearth-ion-doped materials [1-3]. One approach is based on relaxing the constraint of the broad spectrum by combining different crystals. This technique has already given very promising results [4]. Another possibility is the use of atypical crystals combining these two properties [5-8]. Among them, $\mathrm{Yb}^{3+}: \mathrm{CaGdAlO}_{4}$ has been recently proved to be very interesting for the development of diode-pumped short-pulse mode-locked lasers. In fact, this crystal combines two very promising properties. First, it has the broadest and the flattest emission band of all the $\mathrm{Yb}$-doped materials. Second, the measured thermal conductivity of the 2 at. \% Yb:CALGO crystal is as high as $\sim 6.5 \mathrm{~W} \mathrm{~K}^{-1} \mathrm{~m}^{-1}$ [9], allowing high-power pumping. The generation of both very short pulse and high average power femtosecond oscillators has been demonstrated $[8,10]$.

However, for some applications it is interesting to have lower repetition rate (RR) short-pulse oscillators. Actually, the first interest is related to the fact that reducing the $R R$ allows the increase of the energy per pulse for the same average power. This method has been successfully used with Yb:YAG, resulting in the production of $13 \mu \mathrm{J}$ pulses directly at the output of a picosecond oscillator [11,12]. The main challenge, however, especially in the case of ultrafast oscillators, is to maintain the ultrafast duration of the produced pulses at the increased energy level of the low RR cavities. The careful adjustment of the intracavity dispersion is the key factor toward this effort. Additionally, the reduction of the RR is important for oscillators used in chirped-pulseamplification systems. In fact, in these laser chains, the $R R$ is reduced extracavity with acousto- or electro-optic modulators. Starting with a lower RR (therefore less constraint on the rise and fall times of the modulators) allows a lower loss of average power and a more energetic seeding for the amplifiers. Since for a mode-locked laser the $\mathrm{RR}$ is directly related to the cavity round trip, low $\mathrm{RR}$ requires a long cavity of typically $10-100 \mathrm{~m}$. However, generation of short pulses by long cavity oscillators requires special care on the intracavity dispersion management [13] owing to the increased intracavity pulse energy as well as the increased number of beam reflections on the cavity mirrors. This explains the fact that no sub-100 fs pulses have ever been demonstrated for long cavity $(>10 \mathrm{~m})$ with $\mathrm{Yb}$-doped crystals. We are presenting in this Letter the results obtained with Yb:CALGO in extended-cavity lasers. Our goal was the generation of shorter pulses (sub-100 fs) and the assessment of the limitations. During this exploration, we observed a very atypical double-pulse regime with bicolor femtosecond pulses. We also propose an explanation to this regime that, to the best of our knowledge, has never been observed in bulk-material laser oscillators.

The experiment to obtain the high-energy singlepulse regime is performed with a 2\%-doped $\mathrm{Yb}^{3+}$ :CALGO crystal. The crystal is pumped by a $25 \mathrm{~W}$ fiber-coupled diode laser (LIMO) with a $100 \mu \mathrm{m}$

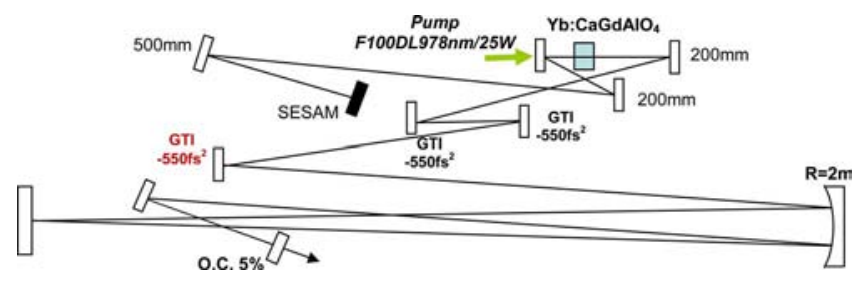

Fig. 1. (Color online) Experimental setup for lowrepetition-rate single-pulse regime. 
core diameter. By the addition of a $q$-preserving telescope, formed by a $2 \mathrm{~m}$ radius of curvature concave mirror and a plane one at a fixed distance of $1 \mathrm{~m}$, we could easily vary the length of the cavity by properly choosing the number of beam bounces on the telescope mirrors $[14,15]$. Initially, the cavity round trip was set at $11 \mathrm{~m}$, corresponding to two reflections on the concave telescope mirror. For the intracavity dispersion compensation we included 3 Gires-Tournois interferometer (GTI) mirrors with a group-velocity dispersion $(\mathrm{GVD})=-550 \mathrm{fs}^{2}$ (Fig. 1). This resulted in a negative net dispersion that can be estimated around $-2600 \mathrm{fs}^{2} /$ round trip (assuming $\sim 50 \mathrm{fs}^{2} / \mathrm{mm}$ for the CALGO crystal and $0 \mathrm{fs}^{2}$ for all unknown dispersion optics: semiconductor saturable absorber mirror (SESAM), output coupler, dichroic mirror). The output coupler transmission was 5\%, which corresponds to an optimum of intracavity power for the chosen GVD compensation. The SESAM (from Amplitude Système) has a modulation depth of $1 \%$ and a saturation fluence $>120 \mu \mathrm{J} \mathrm{cm}^{-2}$.

In this configuration the mode-locked regime was observed for an intracavity pulse energy between 0.3 and $0.5 \mu \mathrm{J}$. The highest average power produced from this mode-locked laser was $650 \mathrm{~mW}$ at $27 \mathrm{MHz}$, corresponding to an output pulse energy of $24 \mathrm{~nJ}$, for $16 \mathrm{~W}$ of pump power. Further increase of the output power resulted in an unstable mode-locking regime leading to $\mathrm{cw}$ operation; no multiple-pulse operation was observed. The pulse duration in this case was measured both with an autocorrelator and a secondharmonic generation (SHG) frequency-resolved optical gating (FROG) setup at $145 \mathrm{fs}$. The spectrum was centered at $1043 \mathrm{~nm}$ and had a bandwidth of $15 \mathrm{~nm}$. The time bandwidth product is 0.6 , and the FROG retrieved spectral phase of the pulse clearly indicates positively chirped pulses with a parabolic phase [Figs. 2(a)-2(c), left side]. These pulses, however, could be still used as direct inputs for various fiber amplifier systems $[16,17]$.

Further increase of the intracavity dispersion to reduce the pulse duration directly out of the cavity made the mode locking of the oscillator impossible. Nevertheless, to compensate this parabolic phase of about $3400 \mathrm{fs}^{2}$, an external compressor based on a standard SF10 prism pair has been used. The compressed pulse duration is $93 \mathrm{fs}$. The corresponding SHG FROG trace and retrieved pulse shape and

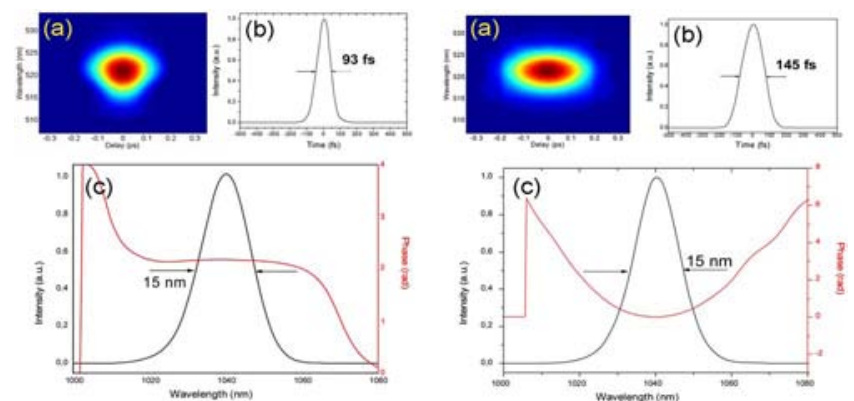

Fig. 2. (Color online) Pulse characterization at the output of the oscillator after (left) and before (right) compression. (a) Experimental SHG FROG trace, (b) retrieved pulse, (c) retrieved spectrum and spectral phase. spectrum are shown in Figs. 2(a)-2(c) (right side). The time bandwidth product is reduced down to $\Delta t \Delta \nu=0.38$ while the main remaining spectral phase distortion is cubic. The values of the remaining phase is $-140 \pm 5 \mathrm{fs}^{2}$ for the quadratic term, $-6000 \pm 500 \mathrm{fs}^{3}$ for the cubic term and $-1.4 \times 10^{6} \pm 4 \times 10^{5} \mathrm{fs}^{4}$ for the fourth order. The energy per pulse after compression is $17 \mathrm{~nJ}$, corresponding to only $70 \%$ compression efficiency as a result of the reduced quality prisms used.

To further reduce the RR, we increased the cavity round trip to $20 \mathrm{~m}$, adding two more round trips inside the telescope. For stable single-pulse mode locking the negative dispersion of the cavity had to be increased by adding two more GTI mirrors of -550 and $-250 \mathrm{fs}^{2}$ (about $-4200 \mathrm{fs}^{2}$ net cavity dispersion). With this setup, the highest average power produced in the single-pulse regime was $240 \mathrm{~mW}$ at $15 \mathrm{MHz}$, corresponding to an output pulse energy of $16 \mathrm{~nJ}$, for $13 \mathrm{~W}$ of pump power. The optimum output coupling of the cavity was $3.8 \%$, corresponding to maximum intracavity pulse energy of $0.42 \mu \mathrm{J}$. Further increase of the energy resulted in multiple-pulse instabilities [17-19]. In Fig. 3 the dependence of the output pulse duration as a function of the average output power is shown. We can clearly observe the onset of double pulsing for output power greater than $250 \mathrm{~mW}$ $(\sim 16.5 \mathrm{~nJ})$. The minimum pulse duration measured in this longer cavity with an autocorrelation and a SHG FROG setup [Figs. 4(a)-4(c)] was $170 \mathrm{fs}$. The spectrum was (for one or two pulses) centered at $1040 \mathrm{~nm}$ and had a bandwidth of $8 \mathrm{~nm}$. The time bandwidth product for this spectrally narrower pulse is 0.37 .

Modifying slightly the $27 \mathrm{MHz}$ setup, it was also possible to observe double-pulse operation. In fact, increasing the intracavity energy and modifying the dispersion (replacing the left-most $-550 \mathrm{fs}^{2}$ GTI in Fig. 1 with another one of $\mathrm{GVD}=-250 \mathrm{fs}^{2}$, reducing the net dispersion to about $-2000 \mathrm{fs}^{2}$ ), the soliton naturally splits in multiple pulses. Multiple-pulse operation is not unusual in femtosecond lasers [18-20], but in our case, owing to the very atypical dispersion curve of the cavity added to the very broad emission spectrum of the $\mathrm{Yb}$ :CALGO, it is possible to generate linearly polarized double pulses with different wavelengths, which we believe is the first demonstration of bicolor femtosecond double-pulse operation. Usually the multiple pulses are separated in time but not in the spectral domain. A very broad spectrum has been obtained with a bandwidth greater than $30 \mathrm{~nm}$ [Fig. 5(a)]. The spectrum can be almost perfectly fit with two Gaussian curves: one centered at $1040 \mathrm{~nm}$

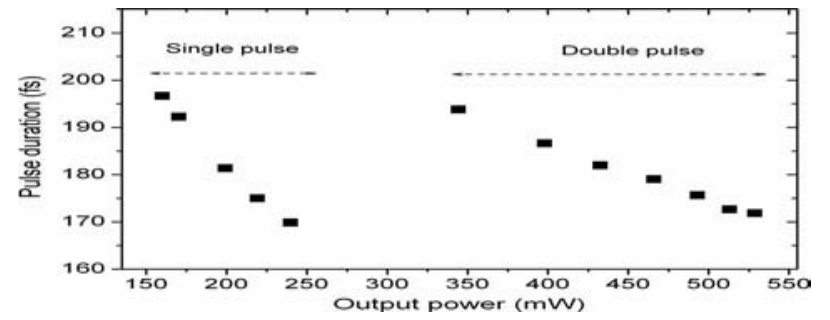

Fig. 3. Pulse duration as a function of the average output power for the $15 \mathrm{MHz}$ laser cavity. 

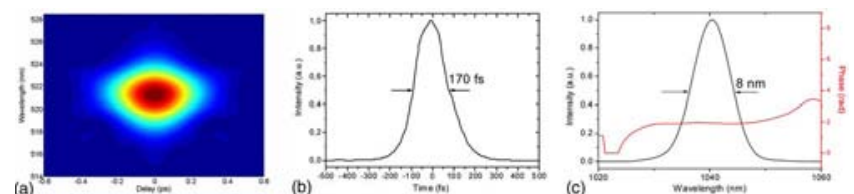

Fig. 4. (Color online) Pulse characterization at the output of the $15 \mathrm{MHz}$ oscillator. (a) Experimental SHG FROG trace, (b) retrieved pulse, (c) retrieved spectrum and spectral phase.

with a bandwidth of $21 \mathrm{~nm}$ and one centered at $1057 \mathrm{~nm}$ with a bandwidth of $10 \mathrm{~nm}$ with lower amplitude $(\sim 60 \%)$. This double pulse is clearly corroborated by the FROG exhibiting four spots forming a diamond shape [Fig. 5(b)]. Although the convergence of the retrieving algorithm from the FROG is low, we can evaluate the pulse durations to be respectively 80 and $130 \mathrm{fs}$ with a pulse separation of $200 \mathrm{fs}$ [Fig. $5(\mathrm{c})]$. This bicolor double-pulse regime might be explained on the basis of the combination of two factors: first, the very broad and constant gain that, in the case of $\mathrm{Yb}$ :CALGO, is inhomogeneous owing to the contribution of its two different crystallographic sites $\mathrm{Gd}^{\mathrm{III}}$ and $\mathrm{Al}^{\mathrm{III}}$, which allows one to reduce cross talk between the two pulses; second, and probably the most important effect, the specific intracavity dispersion distribution resulting in two spectrally distinguished regions of almost constant GVD, overlapping the two Gaussian contributions of the double pulse [inset of Fig 5(a)].

In this Letter we demonstrated that it is possible to obtain sub-100 fs pulse at $27 \mathrm{MHz}$ with a pulse energy of $17 \mathrm{~nJ}$ from an Yb-doped CALGO crystal. Further reduction of the $\mathrm{RR}$ down to $15 \mathrm{MHz}$ has been also achieved, resulting in the generation of $170 \mathrm{fs}$ pulses of $16 \mathrm{~nJ}$ energy directly out of the cavity. We also demonstrated an unprecedented bicolor doublepulse regime within a bulk oscillator generating ultrabroad bandwidth of about $30 \mathrm{~nm}$. Two $\sim 100 \mathrm{fs}$ pulses have been produced temporally separated by $200 \mathrm{fs}$ and spectrally by $17 \mathrm{~nm}$. This bicolor double regime is to our best knowledge an original observation. This regime is possible due to a very specific dispersion curve of the cavity with multiple inflexions,
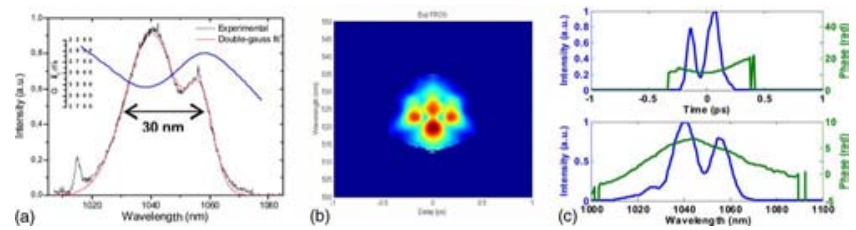

Fig. 5. (Color online) Dual-wavelength double-pulse operation regime. (a) Experimental spectrum and double Gaussian fit (intracavity GTI GVD as inset), (b) SHG FROG trace, (c) SHG FROG retrieved pulse temporalspectral intensity and phase. and this regime is probably helped by the broad and partly inhomogeneous gain of the Yb:CALGO, which tends to reduce cross-talk influence between the bicolor pulses.

\section{References}

1. C. Honninger, F. Morier-Genoud, M. Moser, U. Keller, L. R. Brovelli, and C. Harder, Opt. Lett. 23, 126 (1998).

2. F. Druon, S. Chénais, P. Raybaut, F. Balembois, P. Georges, R. Gaum, G. Aka, B. Viana, S. Mohr, and D. Kopf, Opt. Lett. 27, 197 (2002).

3. F. Druon, S. Chénais, P. Raybaut, F. Balembois, P. Georges, R. Gaumé, P. H. Haumesser, B. Viana, D. Vivien, S. Dhellemmes, V. Ortiz, and C. Larat, Opt. Lett. 27, 1914 (2002).

4. M. Tokurakawa, H. Kurokawa, A. Shirakawa, K. Ueda, H. Yagi, T. Yanagitani, and A. A. Kaminskii, in Advanced Solid-State Photonics, OSA Technical Digest Series (CD) (Optical Society of America, 2008), paper MG2.

5. V. Kisel, A. Troshin, V. Shcherbitsky, N. Kuleshov, V. Matrosov, T. Matrosova, M. Kupchenko, F. Brunner, R. Paschotta, F. Morier-Genoud, and U. Keller, Opt. Lett. 30, 1150 (2005).

6. A. Schmidt, S. Rivier, V. Petrov, U. Griebner, A. Garcia-Cortes, F. Esteban-Betegon, M. D. Serrano, and C. Zaldo, Electron. Lett. 44, 806 (2008).

7. A. Lucca, G. Debourg, M. Jacquemet, F. Druon, F. Balembois, P. Georges, P. Camy, J. Doualan, and R. Moncorgé, Opt. Lett. 29, 2767 (2004).

8. Y. Zaouter, J. Didierjean, F. Balembois, G. LucasLeclin, F. Druon, P. Georges, J. Petit, P. Goldner, and B. Viana, Opt. Lett. 31, 119 (2006).

9. R. Gaumé, B. Viana, D. Vivien, J. P. Roger, and D. Fournier, Appl. Phys. Lett. 83, 1355 (2003).

10. J. Boudeile, F. Druon, M. Hanna, P. Georges, Y. Zaouter, E. Cormier, J. Petit, P. Goldner, and B. Viana, Opt. Lett. 32, 1962 (2007).

11. S. V. Marchese, C. R. Baer, A. G. Engqvist, S. Hashimoto, D. J. Maas, M. Golling, T. Südmeyer, and U. Keller, Opt. Express 16, 6397 (2008).

12. J. Neuhaus, J. Kleinbauer, A. Killi, S. Weiler, D. Sutter, and T. Dekorsy, Opt. Lett. 33, 726 (2008).

13. T. Brabec, C. Spielmann, and F. Krausz, Opt. Lett. 17, 748 (1992).

14. D. Herriott, H. Kogelnik, and R. Kompfner, Appl. Opt. 3, 523 (1964).

15. D. N. Papadopoulos, S. Forget, M. Delaigue, F. Druon, F. Balembois, and P. Georges, Opt. Lett. 28, 1838 (2003).

16. D. N. Papadopoulos, Y. Zaouter, M. Hanna, F. Druon, E. Mottay, E. Cormier, and P. Georges, Opt. Lett. 32, 2520 (2007).

17. D. N. Papadopoulos, I. Martial, M. Hanna, F. Druon, and P. Georges, Opt. Lett. 33, 1431 (2008).

18. V. L. Kalashnikov, E. Sorokin, and I. T. Sorokina, IEEE J. Quantum Electron. 39, 323 (2003).

19. M. Lai, J. Nicholson, and W. Rudolph, Opt. Commun. 142, 45 (1997).

20. M. J. Lederer, B. Luther-Davies, H. H. Tan, C. Jagadish, N. N. Akhmediev, and J. M. Soto-Crespo, J. Opt. Soc. Am. B 16, 895 (1999). 